\title{
A genome-wide screen for Schizosaccharomyces pombe deletion mutants that affect telomere length
}

\author{
Cell Research (2010) 20:963-965. doi:10.1038/cr.2010.107; published online 13 July 2010
}

\section{Dear Editor,}

Both the fission yeast Schizosaccharomyces pombe and the budding yeast Saccharomyces cerevisiae are popular model organisms, and studies using these models have provided many informative clues for solving fundamental biological questions [1], such as DNA replication, cell cycle regulation and gene transcription. Since the completion of genome sequencing of these fungi [2, 3], systematic genetic modification, e.g. gene deletion, has become possible, and genome-wide phenotypic screening for gene function has been widely carried out. For example, Askree et al. and Gatbonton et al. examined the telomere-length change in about 4800 non-essential gene deletion mutants of $S$. cerevisiae, and found that about 250 genes are involved in telomere-length regulation $[4,5]$.

Telomeres are nucleoprotein structures at the ends of linear eukaryotic chromosomes. They help to protect the ends of linear chromosomes from nucleolytic degradation and end-to-end fusions, and provide an effective way to replicate chromosomes completely [6]. Telomeric DNA is composed of highly repetitive sequences. In $S$. pombe, each telomere contains approximately 300 basepairs of a degenerate repeat sequence in which the most frequently occurring motif is TTACAGG, with a consensus sequence of TTAC(A) $(C) \mathrm{G}_{2-8}$ [7]. Some $S$. pombe strains have a homologous telomere-associated sequence (TAS) adjacent to the telomeric-repeat sequence at all six telomeres, whereas other strains contain a TAS in five of six telomeres [8]. Telomeric DNA is usually elongated by a specialized reverse transcriptase, called telomerase, that uses its intrinsic RNA subunit as a template [6]. Fission yeast telomerase is composed of the catalytic subunit Trt1, the RNA template TER1, and an accessory protein subunit Est1 [7]. In addition to telomerase, other proteins and their complexes involved in telomere-length regulation include Poz1, Pot1-Tpz1-Ccq1 complex, Taz1-Rap1 complex [9] and Stn1-Ten1 complex [10]. To systematically search for genes that affect telomeres, we have performed Southern blot analysis to examine telomere-length changes in a collection of 2814 haploid deletion strains of $S$. pombe (see Supplementary information, Data S1). We report here that 168 genes affect the telomere length when they are individually deleted.

The genomic DNA isolated from individual mutant strain was digested with EcoRI restriction enzyme, and hybridized with a TAS1 subtelomeric probe [8]. The terminal restriction fragments of telomeres showed a distinctive band of about $1 \mathrm{~kb}$ on average that contained about $757 \mathrm{bp}$ of subtelomeric repeat and approximately $300 \mathrm{bp}$ of telomeric TTAC(A) $(\mathrm{C}) \mathrm{G}_{2-8}$ repeats (Figure 1) [8]. In addition, there were two major fragments of about 6-8 $\mathrm{kb}$, which might be the specific subtelomeric fragments, representing a unique telomeric restriction pattern of this strain [8]. The band of $3 \mathrm{~kb}$ in Figure 1B detected by a different probe of mis 4 serves as an internal control, because its molecular weight remains constant in various deletion mutants.

To identify the genes that affect telomere length, we have carried out four rounds of screening. In the first round, 831 strains in the deletion mutant set with noticeable telomere-length change were selected. In the second round, 410 deletion mutants were deemed to show reproducible telomere-length change. Then these mutants were retested by at least two additional rounds of reisolating their DNA and doing telomere blots to confirm the telomere-length alteration. After these stringent tests, the number of genes that regulate telomere length was reduced to 168 , including 9 known telomere regulators, as listed in bold in Supplementary information, Table S1. Among these haploid deletion mutants, 17 strains had shorter telomeres, and 151 mutants had longer telomeres compared to wild-type cells. Because many of them exhibited some variation during repeated telomere-blot analyses, the telomere phenotypes were considered from all telomere blot analyses before a gene was categorized to a specific functional group shown in Supplementary information, Table S1. A representative Southern blot from the screen is shown in Figure 1B. The telomere blots for all the deletion strains that have telomere phenotype are provided in the supplementary data (Supple- 
A
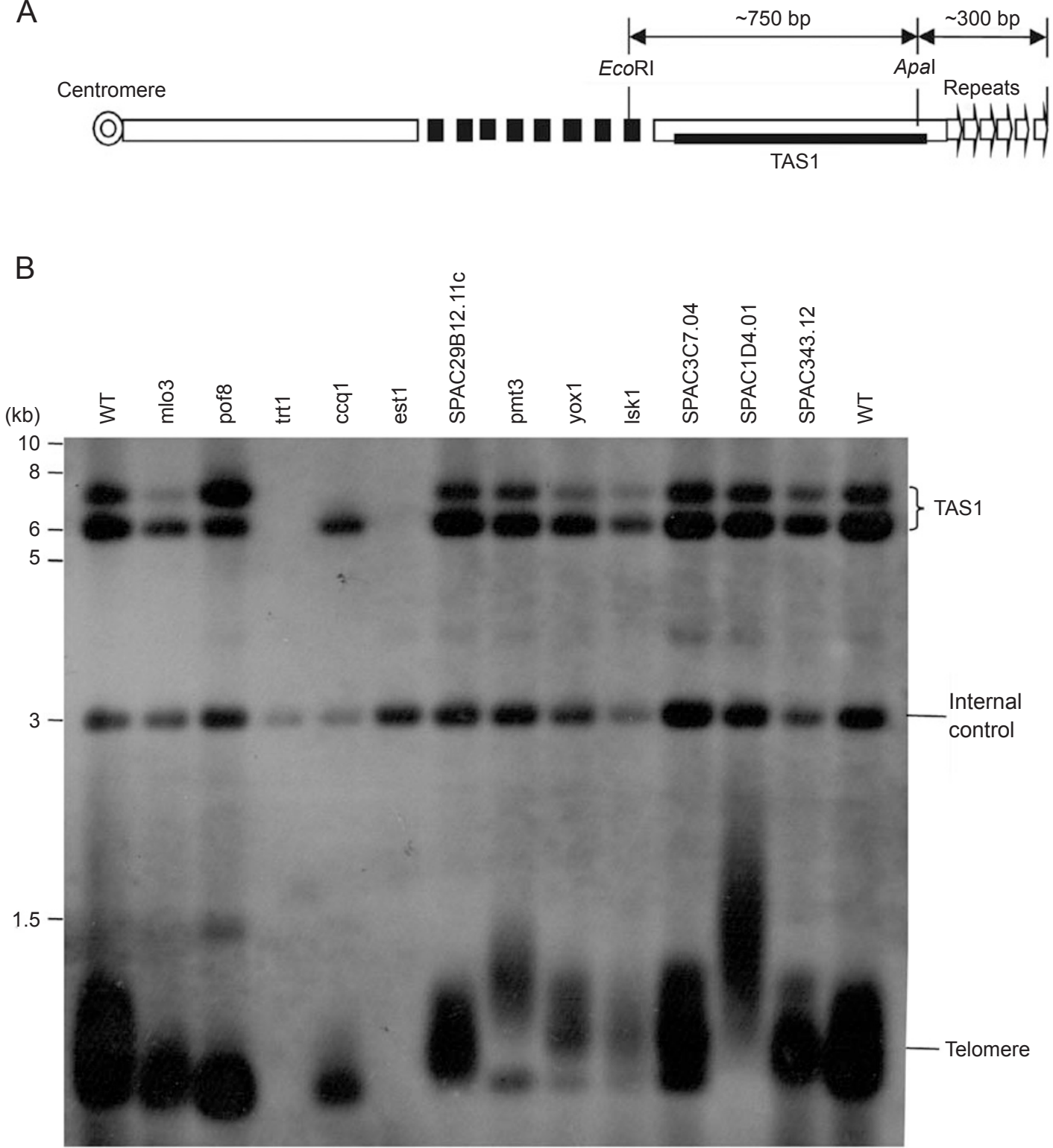

Figure 1 Representative Southern blots of mutants with short and long telomeres. (A) The S. pombe telomeric structure. The terminal telomeric repeat is about $300 \mathrm{bp}$. The TAS1 is used as a probe to visualize the subtelomeric fragment of EcoRI digestion, which is about $1 \mathrm{~kb}$. (B) A representative Southern blot of the deletion mutants. Southern blot of EcoRI-digested DNA of single-gene-knockout mutants of $S$. pombe probed with TAS1.

mentary information, Figure S1). The average telomere length for the deletion mutants was calculated relative to wild-type length, and was grouped into the following categories: slightly short $(<50$ bp shorter than wild type, $\mathrm{SS}$ ); short (50-150 bp, S); very short (> $150 \mathrm{bp}$, VS); slightly long $(<50$ bp longer than wild type, SL); long (50-150 bp, L); and very long (>150 bp, VL) (Supplementary information, Table S1). Surprisingly, a great majority of the 168 mutants exhibited longer than normal telomeres (151 longer versus 17 shorter), which is the opposite of what has been reported in $S$. cerevisiae $[4,5]$. It remains unclear whether this represents a characteristic of telomere regulation in $S$. pombe. Interestingly, 22 deletion mutants, including 9 known telomere regulators, displayed either "very long" or "very short" telomere phenotype (Supplementary information, Table S1). PCRsequencing analysis was performed to verify the identity of the 18 mutants highlighted by red letters in Supplementary information, Table S1 which have significant telomere phenotype. 
Except for some sequence-orphan genes and other conserved hypothetical ones, these genes, as those in $S$. cerevisiae, were categorized into different functional groups based on the Gene Ontology database (listed in Supplementary information, Table S1), including DNA replication/repair/recombination, chromatin modification/remodeling, RNA metabolism and transcription, cell cycle regulation, vesicular traffic, organelle organization and biogenesis, etc. According to previous reports, 29 genes are known to directly regulate telomere length (http://old.genedb.org/genedb/pombe/). Among those, 9 (including est1, trt1, pku80, rap1, rif1, pmt3, poz1, ccq1, bqt1) were recovered in our screening (Supplementary information, Table S1), thus validating our screening; 11 are not in the Bioneer library. The failure to recover the other nine known mutants might be the result of strain background differences or the more stringent criteria we used for assessing the phenotype. In addition to the genes that directly affect telomere maintenance or DNA replication, genes such as pst 2 and $s n f 5$ that are involved in histone modification, chromatin remodeling and nucleosome assembly were also found to affect telomeres (Supplementary information, Table S1). Interestingly, 15 genes of the 168 candidates (as listed in Supplementary information, Table S2), whose deletion changed the telomere length, had their counterparts in S. cerevisiae that have been found to regulate the length of telomeres $[4,5]$. It requires further validation whether these genes directly contribute to telomere maintenance.

In summary, the $S$. pombe Deletion Mutant Library has provided us an opportunity to perform a genomewide screening for genes involved in telomere-length regulation. Our screen has uncovered 159 genes whose roles in telomere regulation have not been reported previously. Our results may provide new clues to how these dynamic pathways integrate to regulate the homeostasis of telomeres.

\section{Acknowledgments}

This work was supported by grant NSFC30770453 to JQZ, and by funding from the Ministry of Science and Technology to LLD.

Ning-Ning Liu ${ }^{1}$, Tian Xu $\mathrm{Han}^{2}$, Li-Lin $\mathrm{Du}^{2}$, Jin-Qiu Zhou ${ }^{1}$
${ }^{I}$ The State Key Laboratory of Molecular Biology, Institute of Biochemistry and Cell Biology, Shanghai Institutes for Biological Sciences, Chinese Academy of Sciences, Graduate School of the Chinese Academy of Sciences, 320 Yue-Yang Road, Shanghai 200031, China; ${ }^{2}$ National Institute of Biological Sciences, Beijing, 7 Science Park Road, Zhong Guan Cun Life Science Park, Beijing 102206, China

Correspondence: Jin-Qiu Zhou ${ }^{\mathrm{a}}, \mathrm{Li}-\mathrm{Lin} \mathrm{Du}^{\mathrm{b}}$

${ }^{\mathrm{a}}$ Tel: +86-21-54921076; Fax: +86-21-54921075

E-mail: jqzhou@sibs.ac.cn

${ }^{\mathrm{b}}$ Tel: +86-10-80726688; Fax: +86-10-80726689

E-mail: dulilin@nibs.ac.cn

\section{References}

1 Dixon SJ, Fedyshyn Y, Koh JL, et al. Significant conservation of synthetic lethal genetic interaction networks between distantly related eukaryotes. Proc Natl Acad Sci USA 2008; 105:16653-16658

2 Goffeau A, Barrell BG, Bussey H, et al. Life with 6000 genes. Science 1996; 274:546, 563-547.

3 Wood V, Gwilliam R, Rajandream MA, et al. The genome sequence of Schizosaccharomyces pombe. Nature 2002; 415:871-880

4 Askree SH, Yehuda T, Smolikov S, et al. A genome-wide screen for Saccharomyces cerevisiae deletion mutants that affect telomere length. Proc Natl Acad Sci USA 2004; 101:86588663 .

5 Gatbonton T, Imbesi M, Nelson M, et al. Telomere length as a quantitative trait: genome-wide survey and genetic mapping of telomere length-control genes in yeast. PLoS Genet 2006; 2:e35.

6 Rog O, Cooper JP. Telomeres in drag: dressing as DNA damage to engage telomerase. Curr Opin Genet Dev 2008; 18:212220.

7 Cooper JP HY. Fission yeast telomeres. In: de Lange T LV, Blackburn EH, eds. Telomeres. 2nd Edition. New York: John Inglis, 2006:495-523.

8 Nakamura TM, Cooper JP, Cech TR. Two modes of survival of fission yeast without telomerase. Science 1998; 282:493-496.

9 Miyoshi T, Kanoh J, Saito M, Ishikawa F. Fission yeast Pot1Tpp1 protects telomeres and regulates telomere length. Science 2008; 320:1341-1344.

10 Martin V, Du LL, Rozenzhak S, Russell P. Protection of telomeres by a conserved Stn1-Ten1 complex. Proc Natl Acad Sci USA 2007; 104:14038-14043.

(Supplementary information is linked to the online version of the paper on Cell Research website.) 